\title{
High glutamate permeability and distal localization of Best1 channel in CA1 hippocampal astrocyte
}

\author{
Hyungju Park ${ }^{1 \dagger}$, Kyung-Seok Han ${ }^{1,2+}$, Soo-Jin Oh', Seonmi Jo ${ }^{1,4}$, Junsung Woo ${ }^{1,2}$, Bo-Eun Yoon ${ }^{1,5}$
} and C Justin Lee $e^{1,2,3^{*}}$

\begin{abstract}
Background: Glutamate is the major neurotransmitter that mediates a principal form of excitatory synaptic transmission in the brain. From the presynaptic terminals of neurons, glutamate is released upon exocytosis of the glutamate-packaged vesicles. In recent years, astrocytes are also known to release glutamate via various routes to modulate synaptic transmission. In particular, we have characterized a glutamate-permeable $\mathrm{Ca}^{2+}$-activated anion channel encoded by Bestrophin 1 gene (Best1) that is responsible for $\mathrm{Ca}^{2+}$-dependent, channel-mediated glutamate release in astrocyte. Best1 channel contains a large pore that is readily permeable to large molecules such as glutamate and GABA. In those studies we obtained permeability ratio of glutamate to $\mathrm{Cl}^{-}$in heterologously expressed mouse Best1 in HEK293T cells and in endogenously expressed mouse Best1 in cultured astrocytes. However, up to now, glutamate permeability of the native Best1 channel in vivo has not been reported.

Findings: In whole-cell recordings of CA1 hippocampal astrocytes, we found that opening of Best1 channel upon activation of a Gq-coupled GPCR, protease-activated receptor 1 (PAR1) generated the anion current carried by glutamate via $\mathrm{Ca}^{2+}$ increase. This $\mathrm{Ca}^{2+}$-evoked glutamate-mediated anion current was unaffected by pretreatment of the inhibitors for a gap junction hemi-channel or $\mathrm{Ca}^{2+}$-activated $\mathrm{K}^{+}$conductance. This astrocytic anion conductance carried by glutamate was mediated by Best1 channel expression in CA1 hippocampal astrocytes, because Best1 knock-down by shRNA expression eliminated astrocytic glutamate conductance by PAR-1 activation. However, we found that these astrocytes showed a deviation in reversal potential of Best1-mediated current from the predicted value. By performing dual patch recording, we concluded that the deviation of reversal potential is due to incomplete space clamping arising from extremely leaky membrane (input resistance ranging 1-3 $M \Omega$ ), very low length constant of astrocytic processes, and the localization of Best1 channel in distal microdomains near synapses. Based on the relative shift of reversal potentials by ion substitutions, we estimated the permeability ratio of glutamate and $\mathrm{Cl}^{-}\left(\mathrm{P}_{\text {glutamate }} / \mathrm{P}_{\mathrm{Cl}}\right)$ as 0.53 .
\end{abstract}

Conclusions: Our study shows that Best1, located at the microdomains near the synaptic junctions, has a significantly high permeability to glutamate in vivo, serving as the prominent glutamate-releasing channel in astrocytes, mediating the release of various gliotransmitters in the brain, and playing an important role in modulating synaptic transmission.

Keywords: Astrocyte, Bestrophin-1, Glutamate, Anion channel

\footnotetext{
* Correspondence: cjl@kist.re.kr

${ }^{\dagger}$ Equal contributors

${ }^{1}$ Center for Neural Science and WCI Center for Functional Connectomics,

Korea Institute of Science and Technology (KIST), Seoul, Korea

${ }^{2}$ Neuroscience Program, University of Science and Technology (UST),

Daejeon, Korea

Full list of author information is available at the end of the article
} 


\section{Background}

Astrocytes modulate neuronal function by releasing glutamate in response to neuronal activity $[1,2]$. Astrocytically released glutamate has been shown to activate neighboring neuronal glutamate receptors such as $\mathrm{N}$ methyl-D-aspartic acid receptor (NMDAR) which is a critical player for diverse neuronal function [3-5]. Thus, delineation of underlying mechanisms of glutamate release from astrocytes has received much attention.

Recently, it has been reported that astrocytic glutamate is released through glutamate-permeable channels, such as $\mathrm{Ca}^{2+}$-activated anion channel, Best1 and TREK-1 containing two pore potassium channel $[4,5]$. In particular, Best1 is responsible for the $\mathrm{Ca}^{2+}$-dependent, channelmediated glutamate release from astrocytes [4]. Due to the preferential subcellular localization of Best1 at the microdomain near neuronal synaptic sites [4], it is believed that glutamate released through Best1 specifically targets postsynaptically localized neuronal NMDA receptors [3] and plays a critical role in modulating synaptic function [3].

The unique property of Best1 in mediating channelmediated glutamate release originates from its pore that allows large ions and molecules to pass through [6,7]. Best1 has been shown to be permeable to large ions and molecules with a significant permeability ratio of anion to $\mathrm{Cl}^{-}\left(\mathrm{P}_{\mathrm{x}} / \mathrm{P}_{\mathrm{Cl}}\right)$ : GABA (0.19) [6], isothionate (0.47) [7], bicarbonate (0.44) [8], gluconate (0.4) [9]. Despite the strong evidence for functional expression, physiological importance, and unique biophysical property of Best1, there has been no description or estimation of glutamate permeability of Best1 in vivo [4,7]. To address this issue, we performed an electrophysiological measurement of whole-cell anion conductance from astrocytes in acute hippocampal slice to identify a $\mathrm{Ca}^{2+}$-dependent glutamate conductance mediated by Best1.

\section{Results and discussions}

Characterization of Best1-mediated glutamate conductance from hippocampal CA1 astrocytes

To identify the anion channel-mediated glutamate conductance from astrocytes in hippocampal slices, we utilized PAR-1 as a tool for eliciting $\mathrm{Ca}^{2+}$-dependent anion conductance from hippocampal astrocytes. Previously, it has been demonstrated that both in cultured astrocytes and astrocytes in hippocampal slice, PAR- 1 activation by treatment of its agonist, TFLLR- $\mathrm{NH}_{2}$ peptide $(30 \mu \mathrm{M}$ of TFLLR), can induce $\mathrm{Ca}^{2+}$-activated anion current (CAAC), and this anion conductance is mediated by Best1 channel $[4,7]$. We tested whether Best1-dependent anion conductance is carried by glutamate. A whole-cell patch clamp was made onto hippocampal CA1 astrocytes labeled with sulforhodamine-101 (SR-101) [7]. To reduce the voltageclamp error due to electrical coupling among neighboring astrocytes through gap junction [10], we performed the experiment with the slices pretreated with a gap junction blocker, $100 \mu \mathrm{M}$ carbenoxolone for at least 30 minutes. $\mathrm{Cl}^{-}$, glutamate, or gluconate was included as a predominant anion of the pipette internal solution as previously described $[7,11]$ to compare the permeability of each anion. The changes in conductance were monitored by a series of periodic voltage ramps from $+100 \mathrm{mV}$ to $-100 \mathrm{mV}$ applied before and during TFLLR application (Figure 1A, B) and by subtracting ramp currents recorded during TFLLR application from those measured before TFLLR application to obtain the I-V relationship of the TFLLR-induced CAAC (Figure 1C). The normalized I-V relationships from our recordings showed that substituting glutamate and gluconate with the intracellular $\mathrm{Cl}^{-}$resulted in a significant inward current at the holding potential of $-100 \mathrm{mV}$ (Figure 1D), indicating a significant efflux of glutamate and gluconate at this potential [12].

We next tested whether this astrocytic glutamate conductance is mediated by Best1. In our previous report, we demonstrated that over $90 \%$ of GFAP positive astrocytes in stratum radiatum of CA1 hippocampus express functional Best1, and this Best1 mediates the TFLLRinduced CAAC [7]. Thus, it is plausible to hypothesize that Best1 is also responsible for glutamate-mediated conductance elicited by PAR-1 activation. To reduce the Best1 expression in the astrocytes, we utilized the Best1sensitive short hairpin RNA (shRNA) [7]. A whole-cell patch clamp was made onto the astrocytes expressing scrambled- or Best1-shRNA (identified by co-staining of SR-101 and GFP (co-expressed with shRNA)) using the pipette solution containing glutamate as a main anion (Figure 1E). We found that TFLLR-induced anion conductance in Best1-shRNA expressing astrocytes was almost completely eliminated, compared to scrambled-shRNA expressing astrocytes (Figure 1E, F). The effect on TFLLRinduced glutamate efflux was quantified by measuring current magnitude at $-100 \mathrm{mV}$ : glutamate efflux at this potential was significantly smaller in astrocytes from mice injected with Best1-shRNA than with scrambled-shRNA or from naïve mice (Figure 1F). Taken together, our data indicate that Best1 in hippocampal astrocytes is required for generating TFLLR-induce anion conductance carried by glutamate.

\section{Astrocytic glutamate conductance is unaffected by gap junction hemichannel or $\mathrm{Ca}^{2+}$-activated $\mathrm{K}^{+}$channel}

We performed sets of control experiments to test whether Best1-dependent glutamate conductance is a genuine glutamate and to eliminate possible contributions of other channels to the TFLLR-induced conductance. Because of the abundant expression of gap-junctions between astrocytes and its possible contribution to our measurement, 


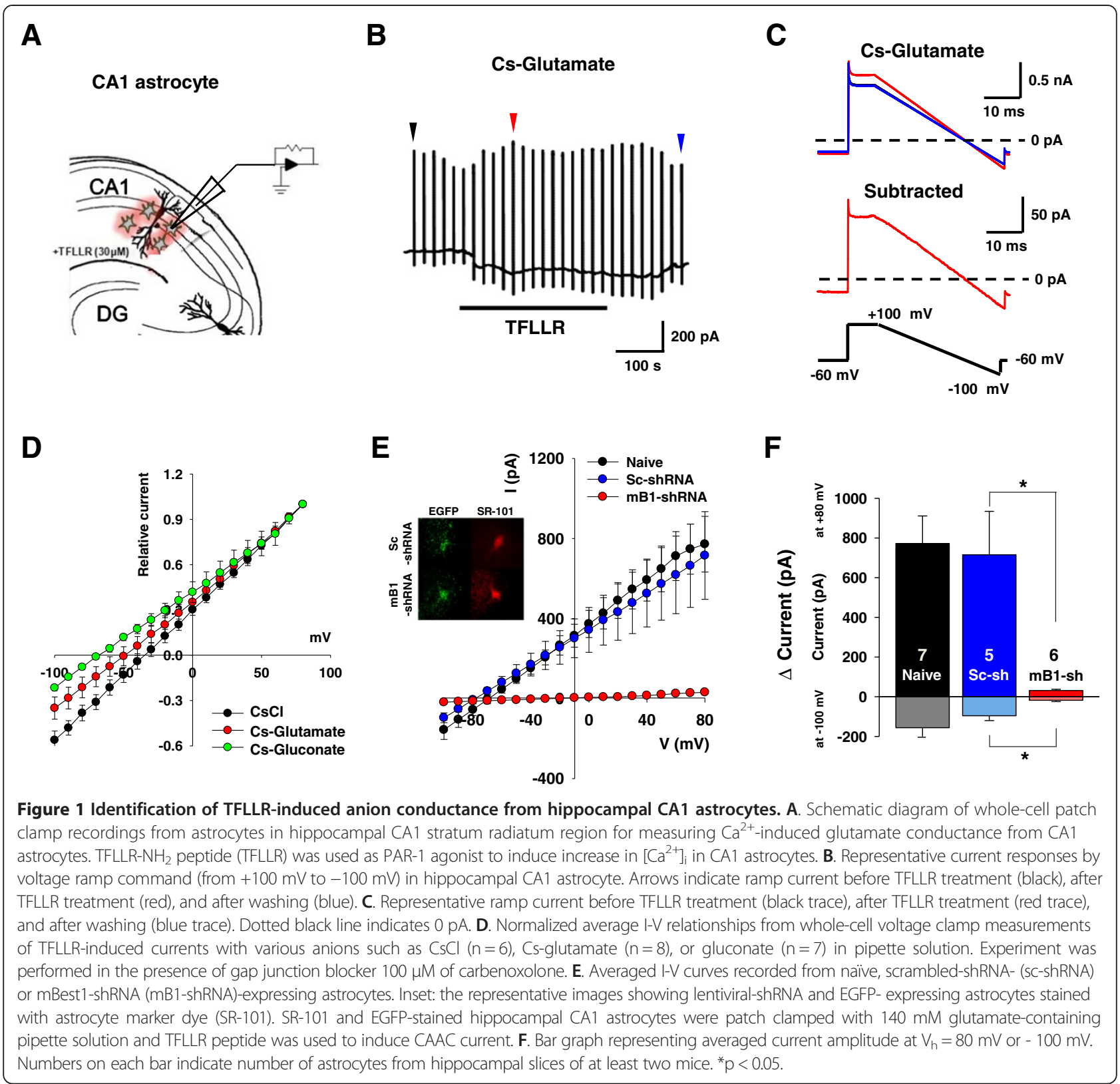

we compared TFLLR-induced glutamate conductance in the presence or absence of carbenexolone (CBX; $100 \mu \mathrm{M})$. It has been known that CBX blocks electrical signal through gap junction in astrocytes [13-16]. Firstly, we measured Rm value of hippocampal astrotytes in the presence or absence of CBX. Rm value was slightly increased from 9.56 $\pm 1.814 \mathrm{M} \Omega$ (mean \pm s.e.m; $\mathrm{N}=10$; in the absence of $\mathrm{CBX})$ to $14.77 \pm 2.74 \mathrm{M} \Omega(\mathrm{N}=9$; in the presence of $\mathrm{CBX}$ ) by CBX treatment, but this change was not significant ( $\mathrm{p}=0.1250$, unpaired $\mathrm{t}$-test, data not shown). This insignificant change in the membrane resistance by CBX treatment is probably due to the astrocytes' leaky property (usually $\mathrm{Rm}$ is about 10 mega ohm), resulting in the length constant-mediated voltage clamping error. Furthermore, our data showed that there is no gap-junction dependent change in glutamate conductance in our experimental condition (Figure 2A, B) indicating no role of gap-junction mediated mechanism in generating astrocytic glutamate conductance.

Next, it is still possible that $\mathrm{Ca}^{2+}$-activated $\mathrm{K}^{+}$channel contributes to the TFLLR-induced conductance from astrocytes. The major cation in the pipette solution is $\mathrm{Cs}^{+}$ which is known to inhibit the most of current mediated by $\mathrm{Ca}^{2+}$-activated $\mathrm{K}^{+}$channel, SK1 channel [17]. To directly test whether $\mathrm{Cs}^{+}$based internal pipette solution blocks $\mathrm{Ca}^{2+}$ activated $\mathrm{K}^{+}$channel, and to show that $\mathrm{Ca}^{2+}$-activated 

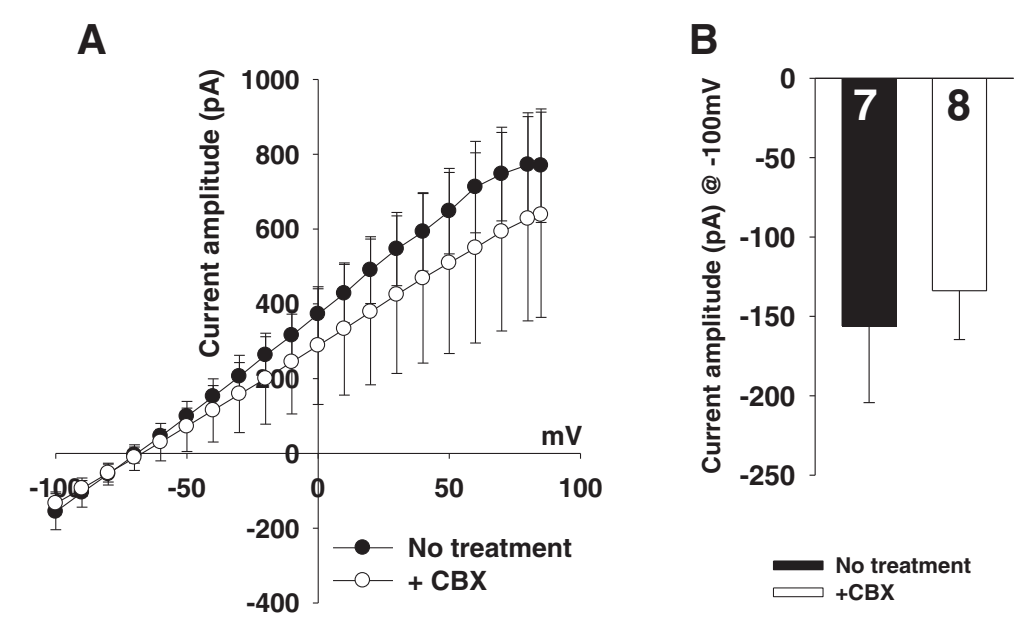

Figure 2 TFLLR-induced conductance is independent on activation of gap-junction hemichannels and $\mathrm{Ca}^{2+}$ activated potassium channel. A. Averaged current-voltage relationships from TFLLR-induced current using Cs-Glutamate pipette solution in the presence of gap junction blocker $(+\mathrm{CBX})$ or normal ACSF bath solution (No treatment). B. Bar graphs indicates the averaged current amplitude at holding potential of $-100 \mathrm{mV}$ (Current amplitude @-100 mV) to test whether glutamate-mediated current in astrocytes is reduced by inhibiting the gap junction activity. $p=0.7$ for Current amplitude @ -100 mV; unpaired t-test.

$\mathrm{K}^{+}$conductance does not contribute to TFLLR-induced conductance, we expressed SK1 channel in HEK293T cells and measured the SK1-mediated whole-cell current induced by $\mathrm{Ca}^{2+}$-included pipette solution. When the major cation was $\mathrm{K}^{+}$ion in the pipette solution, $\mathrm{Ca}^{2+}$-induced $\mathrm{K}^{+}$ conductance was rapidly evoked upon whole-cell rupture and slowly desensitized, but still remained even several minutes after the whole-cell rupture (Figure 3A, B). By contrast, when $\mathrm{Cs}^{+}$-containing pipette solution was used, $\mathrm{Ca}^{2+}$-induced $\mathrm{K}^{+}$conductance by whole-cell rupture of SK1 expressing HEK293T cell was disappeared within $~ 60 \mathrm{sec}$ (Figure 3C, D), eliminating the possible contribution of $\mathrm{Ca}^{2+}$-induced $\mathrm{K}^{+}$conductance to our TFLLR-induced glutamate current. This is consistent with our previous study showing that the size of TFLLR-induced CAAC currents recorded using $\mathrm{K}^{+}$based pipette solution is similar with that using $\mathrm{Cs}^{+}[7]$.

\section{Dual patch-clamp recording from a single astrocyte reveals voltage error}

To determine the glutamate permeability ratio, we examined the reversal potential from the I-V relationships that we obtained in Figure 1B. Surprisingly, the observed reversal potential of the intracellular $\mathrm{Cl}^{-}$condition $(-34.8 \mathrm{mV})$ deviated significantly from the predicted $0 \mathrm{mV}$ according to the Nernst equation under symmetrical $\mathrm{Cl}^{-}$. This was markedly different from the reversal potential obtained from Bergmann glial cells under similar recording and symmetrical $\mathrm{Cl}$ - condition [6]: the reversal potential of the Best1-mediated CAAC current from Bergmann glial cell was similar to the predicted value of $0 \mathrm{mV}$ (Figure 4F) [6]. The reversal potential was clearly shifted by substitution of intracellular anions [shifted from $-34.8 \mathrm{mV}\left(\mathrm{Cl}^{-}\right)$to $-49.1 \mathrm{mV}$ (glutamate) and $-68.1 \mathrm{mV}$ (gluconate)] (Figure 1B). The shift in reversal potential by ion substitutions indicates that glutamate is less permeable than $\mathrm{Cl}^{-}$but more permeable than gluconate, as predicted by the size of the anions [12]. The deviation in the observed reversal potential from the symmetrical $\mathrm{Cl}^{-}$condition raised a possibility that voltage errors can be substantial under conventional single-electrode patch-clamp configuration due to astrocyte's inherent low membrane resistance [10].

To estimate the degree of voltage error due to astrocyte's leaky membrane in our experimental condition, we next performed a dual patch-clamp recording onto CA1 astrocytes to observe the actual membrane voltage during the voltage clamp experiment. Experimentally, two recording electrodes were sealed consecutively to a single astrocytic soma (Figure 5A). One electrode was used to perform whole-cell voltage clamp recording, and the other to monitor the actual voltage change in current clamp mode without adding holding currents. Firstly, we recorded passive conductance during a series of step-voltage protocol in astrocytes (Figure 5B). We observed a significant discrepancy between the command voltage and the actual voltage (50\% error at command voltage of $+40 \mathrm{mV}$, Figure $5 \mathrm{~B}$ ), consistent with the previous report [10]. This discrepancy is mostly likely due to a voltage drop across the access resistance of the patch pipette, which is shown to be prominent especially when the membrane resistance is lower than the access resistance as in astrocyte [10]. However, this voltage error due to a voltage drop across the access resistance should not and did not 


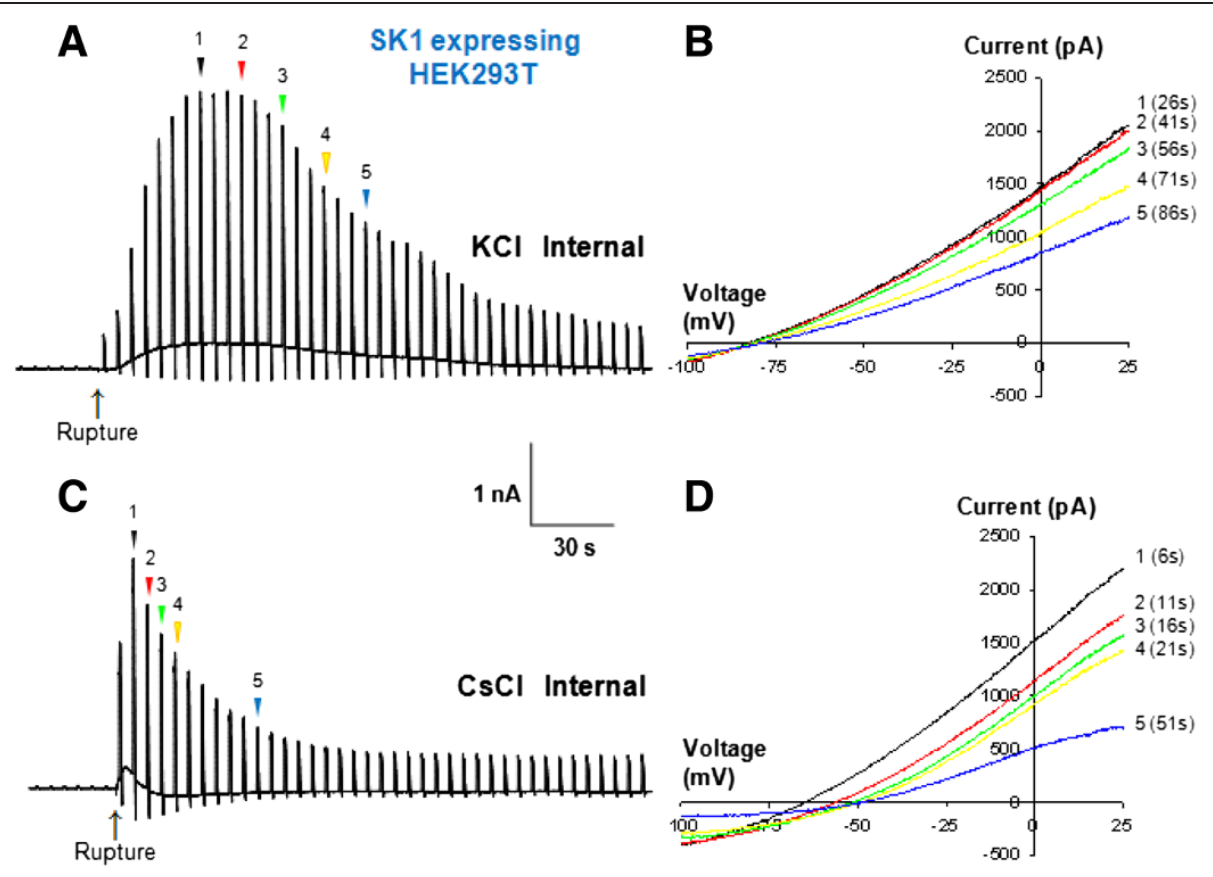

Figure 3 TFLLR-induced conductance is mediated by Best1 channel in hippocampal CA1 astrocytes. A. Representative current responses induced by whole-cell patch clamp from HEK293T expressing hSK1 using KCl-containing pipette solution $(n=8)$. The current responses were recorded in response to a voltage ramp command (from -100 to $+100 \mathrm{mV}, 1 \mathrm{~s}$ duration, $0.2 \mathrm{~Hz} ; V_{\mathrm{h}}$ of $-60 \mathrm{mV}$. Internal pipette solution is composed of (mM) $150 \mathrm{KCl}, 10 \mathrm{HEPES}, 1 \mathrm{CaCl}_{2}, 1 \mathrm{MgCl}_{2}$, and $5 \mathrm{EGTA}\left(100 \mathrm{nM}\right.$ free $\left.\mathrm{Ca}^{2+}\right)$. B. The 5 selected representative current-voltage relationships recorded from a). C. Representative current responses induced by whole-cell patch clamp from HEK293T expressing hSK1 using KCl-containing pipette solution $(n=7)$. D. The 5 selected representative current-voltage relationships recorded from c). Averaged time that Er reaches to plateu from rupture is $63.4 \pm 14.6 \mathrm{~s}(\mathrm{n}=7)$.

affect the reversal potential of the passive conductance, which falls near the equilibrium potential for $\mathrm{K}^{+}$ion at around $-80 \mathrm{mV}$ (Figure 5B) [10].

We then performed dual patch-clamp recording to isolate the CAAC current induced directly by $\mathrm{Ca}^{2+}$ in the patch pipette solution and by using the blocker, 5Nitro-2-(3-phenylpropylamino)benzoic acid (NPPB) to obtain the NPPB-sensitive CAAC current under symmetrical $\mathrm{Cl}^{-}$condition from a single astrocyte (Figure 5). This allowed us to isolate Best1-mediated conductance during the dual patch clamp recording and to confirm whether NPPB-sensitive current in this condition is same with the one recorded in Figure 1A. We isolated NPPB-sensitive current using voltage ramp protocol from $+100 \mathrm{mV}$ to $-100 \mathrm{mV}$ (Figure $5 \mathrm{C}$ ) and plotted as an $\mathrm{I}-\mathrm{V}$ relationship (Figure 5D). Instead of using the command voltage for the plot, we used the recorded actual voltage for the I-V relationship to bypass the voltage error due to access resistance (Figure 5D). The I-V relationship of Best1-medaited, NPPB-sensitive current showed a reversal potential of around $-40 \mathrm{mV}$ (Figure 5D), which was similar to the deviation that we observed with the reversal potential of TFLLR-induced CAAC current in Figure 1B $(-34.8 \mathrm{mV})$.
Deviation of reversal potential due to specific localization of Best1 in microdomain

What is the source of $-40 \mathrm{mV}$ deviation in the reversal potential of both NPPB sensitive current and TFLLR-induced CAAC current? The distortion of voltagegated currents due to incomplete space-clamp in neuronal dendrites under patch-clamp technique has been extensively reported [18-25]. Unlike neurons, astrocytes are known to exhibit high expression of background potassium channels, which results in very low membrane resistance in a unit length, $\mathrm{R}_{\mathrm{m}}$. In addition, astrocytes contain elaborate processes with a small diameter of around $1 \mu \mathrm{m}$, which should result in high axial resistance per unit length, $\mathrm{R}_{\mathrm{i}}$. The combination of these two factors affects the length constant by the relationship, $\lambda=\sqrt{\frac{R m}{R i}}$, which governs the degree of voltage error $(\Delta \mathrm{V})$ due to an incomplete space clamp in voltage clamping experiments by the relationship, $\Delta V(X)=V_{\max }\left(e^{-x / \lambda}\right)$, where $X$ represents the distance from the recording electrode. The length constant $\lambda$ can be rewritten as, $\lambda=\sqrt{\frac{R m}{p}} \times \frac{a}{2}$, where $\rho$ is the specific resistivity of astrocytic process and $a$ is the diameter of astrocytic process. In case of hippocampal astrocytes, $\lambda$ should be very low due to low $R_{m}$ and high $R_{i}$ and 


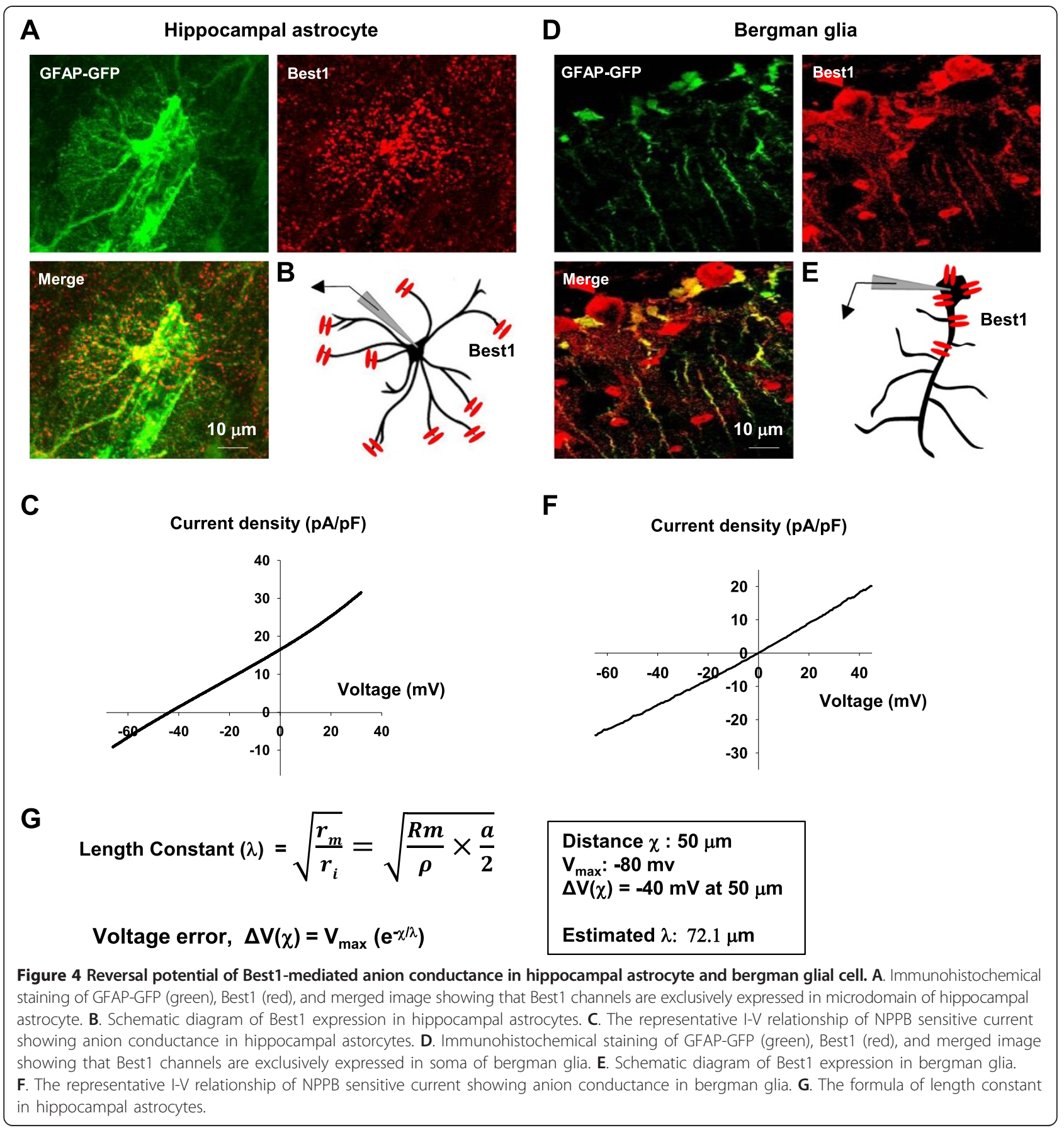

therefore the voltage error, $\Delta \mathrm{V}$ should be significantly high. Similar to an axon of mammalian neuron, the diameter of each astrocytic process is estimated to be around $1 \mu \mathrm{m}$ [26]. Best1 is reported to be expressed mostly in the distal microdomains of astrocytes, near synaptic junctions, as shown in our previous study [4] as well as in Figure 4A. Thus, the average distance of Best1 from the recording electrode, $X$, should be around $50 \mu \mathrm{m}$ (Figure 4B). Under symmetrical $\mathrm{Cl}^{-}$condition, the expected voltage at the microdomain when the Best1 is fully open should be around $0 \mathrm{mV}$. Thus, the $\mathrm{V}_{\max }$ can be estimated to be around $-80 \mathrm{mV}\left(\mathrm{V}_{\max }=-80 \mathrm{mV}-0 \mathrm{mV}\right)$, based on the fact that the resting membrane potential at soma around the recording electrode is around $-80 \mathrm{mV}$. Using the observed voltage error of $-40 \mathrm{mV}$ for $\Delta \mathrm{V}(\mathrm{X})$ at $50 \mu \mathrm{m}$, we can now estimate the length constant $\lambda$ for astrocytic processes to be $72.1 \mu \mathrm{m}$ (or $0.0721 \mathrm{~mm}$ ) (Figure 4G), which is much lower (44 fold less) than the reported length 


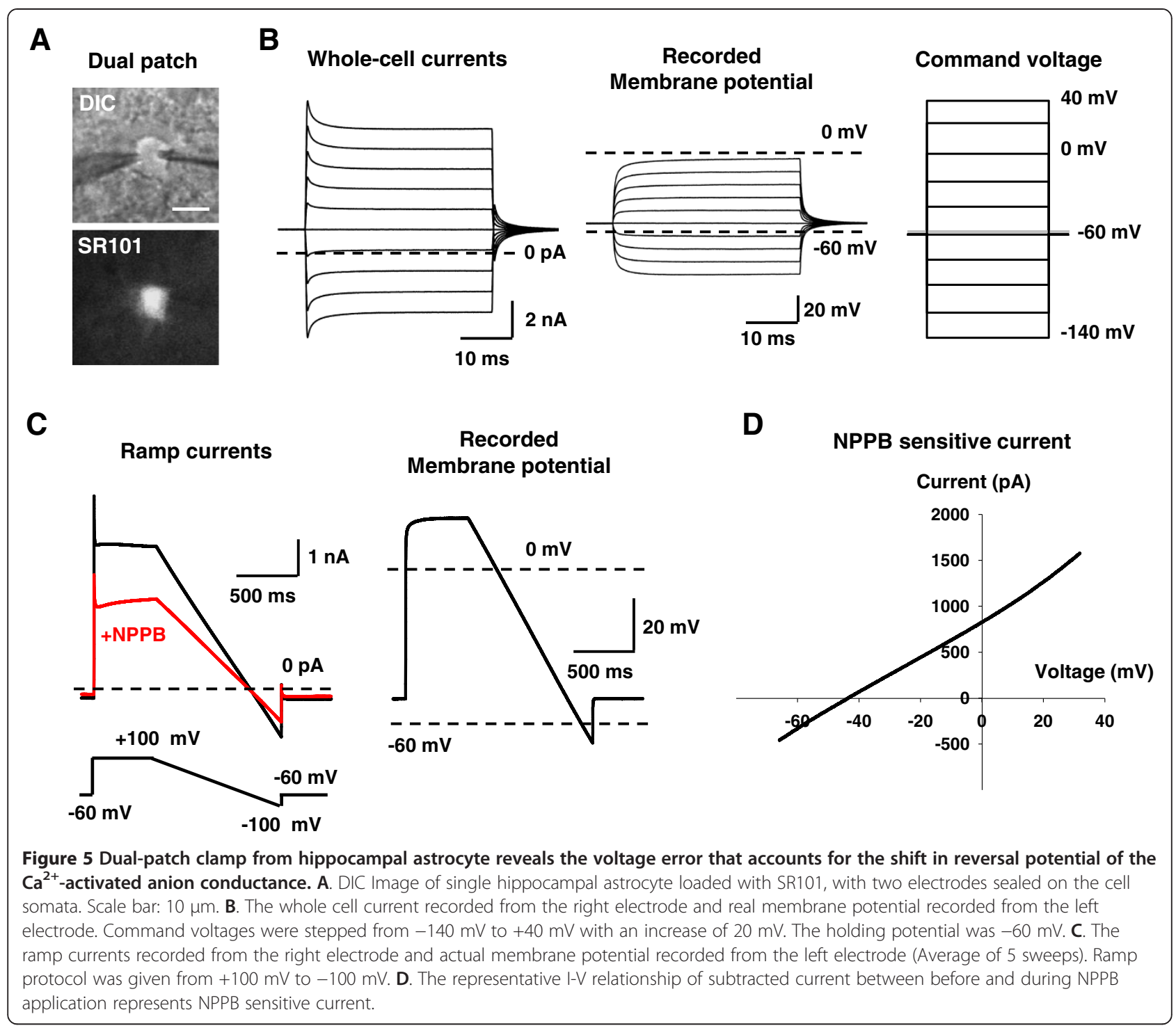

constant for neuronal dendrite $(\lambda=3.16 \mathrm{~mm}$, [27]). Unlike hippocampal astrocytes, Bergmann glial cells express Best1 in the cell body as well as main processes (Figure 4D) [6]. Therefore, the voltage error is minimal due to minimal distance between the locations of Best1 to the recording electrode. This is most likely the reason for the consistency between the observed reversal potential and the predicted value in Bergmann glial cells (Figure 4D). Taken together, we concluded that the I-V relationships in Figure $1 \mathrm{~B}$ are deviated by about $-40 \mathrm{mV}$ due to voltage error arising from incomplete space-clamp, which originates from the very low length constant of the astrocytic processes. These results and estimations strongly support the idea that functional Best1 channel is mostly expressed in distal microdomains of hippocampal astrocytes, as predicted by the immunohistochemical and electrophysiological evidence.

\section{Calculation of glutamate permeability ratio}

The relative shift in the reversal potential might underestimate the true permeability ratio due to the evident voltage error. Nevertheless, we found a shift in reversal potential for each anion in accord with anion substitution in the pipette solution (Figure 1B). Based on the shift in reversal potential, it was possible to calculate a relative permeability of each anion to $\mathrm{Cl}^{-}$anion according to the Goldman-Hodgkin-Kats equation (see Materials and Methods). The estimated permeability ratio of glutamate to $\mathrm{Cl}^{-}$was $\mathrm{P}_{\text {glutamate }} / \mathrm{P}_{\mathrm{Cl}}=0.53$, which was in the range of the reported values between 0.47 for cultured astrocytes [7] and 0.67 for heterologously expressed Best1 channels in HEK293T cells [4]. The calculated glutamate permeability ratio of Best1 was much higher than the reported GABA permeability ratio, $\mathrm{P}_{\mathrm{GABA}} / \mathrm{P}_{\mathrm{Cl}}=0.19$, of the Best 1 in Bergmann glial cells of the cerebellum [6]. 


\section{Conclusion}

In conclusion, our results indicate that Best1 in CA1 hippocampal astrocytes shows a relatively high permeability to glutamate. In addition, its specific localization at microdomain near synaptic junctions is further evidenced by the deviation of the reversal potential of Best1-mediated CAAC current. This unique biophysical property of Best1 makes this channel a prominent molecule responsible for channel-mediated release of important gliotransmitters such as glutamate and GABA. It is possible that Best1 channel is also permeable to many other important gliotransmitters such as D-serine, aspartate and taurine. Future studies will provide these intriguing possibilities of their roles in channel-mediated gliotransmitter release and in synaptic functions.

\section{Methods}

\section{Cell culture and electrophysiology}

HEK293T cell culture was performed as previously described [3]. For measuring SK1 conductance, HEK293 cells were transfected with human SK1-containing plasmid pcDNA3.1-hSK1 using Effectene (Qiagen, Valencia, CA, USA) at $1 \mathrm{mg}$ of DNA per $35-\mathrm{mm}$ culture dish. Transfected HEK293 cells were recorded using a conventional whole-cell patch-clamp within 48 hrs. Fire-polished borosilicate glass patch pipettes were $3 \sim 5 \mathrm{M} \Omega$. Experiments were conducted at room temperature $\left(20 \sim 25^{\circ} \mathrm{C}\right)$. Current-voltage curves were established by applying $500 \mathrm{~ms}$ duration voltage ramps from -100 to $+100 \mathrm{mV}$ $\left(V_{h}=-70 \mathrm{mV}\right)$. Data were acquired with an Axopatch 200A amplifier controlled by Clampex 10.0 software via a Digidata 1322A data acquisition system (Molecular Devices, Sunnyvale, CA, USA). The extracellular solution is composed of $(\mathrm{mM}) 150 \mathrm{NaCl}, 10$ HEPES, $3 \mathrm{KCl}, 2$ $\mathrm{CaCl} 2,2 \mathrm{MgCl} 2$, and 5.5 glucose at $\mathrm{pH} 7.3$ adjusted with $\mathrm{NaOH}$ (300 320 mOsm). Internal pipette solution is composed of $(\mathrm{mM}) 106 \mathrm{CsCl}$ or $\mathrm{KCl}, 20 \mathrm{TEA}-\mathrm{Cl}, 10 \mathrm{BAPTA}$, 8.7 $\mathrm{CaCl}_{2}, 0.5 \mathrm{MgCl}_{2}$, and 5 HEPES (500 $\mathrm{nM}$ free $\mathrm{Ca}^{2+}$ ).

\section{Production of mBest1-shRNA containing lentivirus and delivery into mouse hippocampus}

Scrambled shRNA and $m$ Best1-shRNA were inserted into pSicoR lentiviral vector (provided by Dr. T. Jacks through Addgene Inc.) [28] as previously described [27]. For shRNA expression into hippocampal CA1 region, lentivirus (produced by Macrogen, Korea) was delivered into hippocampal CA1 region of wild type C57BL/6 mice by a stereotaxic surgery method [29].

\section{Slice preparation and electrophysiology}

Horizontal or transverse mouse brain slices $(300 \sim 400 \mu \mathrm{m})$ containing hippocampus were acutely prepared as previously described [3]. SR-101 (Sigma; $1 \mu \mathrm{M}$ ) dye was loaded into acute brain slice as previously described [30]. Prepared slices were left to recover for at least $1 \mathrm{~h}$ before recording in oxygenated $\left(95 \% \mathrm{O}_{2}\right.$ and $\left.5 \% \mathrm{CO}_{2}\right)$ artificial cerebrospinal fluid (ACSF; in mM, $130 \mathrm{NaCl}, 24 \mathrm{NaHCO}_{3}, 3.5 \mathrm{KCl}, 1.25$ $\mathrm{NaH}_{2} \mathrm{PO}_{4}, 1 \mathrm{CaCl}_{2}, 3 \mathrm{MgCl}_{2}$ and 10 glucose, $\mathrm{pH}$ 7.4.; room temperature). The standard ACSF recording solution was composed of (mM): $130 \mathrm{NaCl}, 24 \mathrm{NaHCO}_{3}, 3.5 \mathrm{KCl}, 1.25$ $\mathrm{NaH}_{2} \mathrm{PO}_{4}, 1.5 \mathrm{CaCl}_{2}, 1.5 \mathrm{MgCl}_{2}$ and 10 glucose saturated with $95 \% \mathrm{O}_{2}-5 \% \mathrm{CO}_{2}$, at $\mathrm{pH} 7.4$. To observe $\mathrm{Ca}^{2+}$-induced glutamate conductance in CA1 astrocytes, the pipette solution contained (in mM) 133 Cs-glutamate, $1 \mathrm{MgCl}_{2}$, and 10 HEPES, at pH 7.3 adjusted with $\mathrm{CsOH}$ ( $280 \mathrm{Osm})$. For $\mathrm{Cl}^{-}$or gluconate-based internal solution, Cs-glutamate was replaced with the same concentration of $\mathrm{CsCl}$ or Csgluconate. To block the effect of neuronal spontaneous activity on astrocytes, TTX $(0.5 \mu \mathrm{M}$; Tocris $)$ was added into ACSF. Experiments with a holding current more than $-100 \mathrm{pA}$ or a change in input resistance $>30 \%$ of control were rejected. To induce CAAC activation from CA1 astrocytes, $30 \mu \mathrm{M}$ of TFLLR was bath-treated. In one series of experiments, dual-patch recording from a single astrocyte was used to directly measure the voltage errors in whole-cell voltage clamp recording. The standard electrode solution contained the following (in $\mathrm{mM}$ ): $140 \mathrm{KCl}, 0.5 \mathrm{CaCl}_{2}, 1.0 \mathrm{MgCl}_{2}$, 5 EGTA, 10 HEPES, $3 \mathrm{Mg}$-ATP, and $0.3 \mathrm{Na}-\mathrm{GTP}$ (pH 7.3). The $\mathrm{pH}$ was adjusted to 7.3 with $\mathrm{KOH}$.

Length constant $(\lambda)=\sqrt{\frac{R m}{R i}}(\mathrm{Rm}$ is membrane resistance, and $\mathrm{Ri}$ is axial resistance.).

$\mathrm{V}(\mathrm{x})=\mathrm{Vmax}\left(\mathrm{e}^{-\mathrm{x} / \lambda}\right)(\mathrm{x}$ is the distance from the start of the potential).

\section{Immunohistochemistry}

Rabbit polyclonal Best1 IgG was produced using antigen (Ab Frontier). Primary antibody was then applied at appropriate dilution [Chicken polyclonal GFP at 1:1000 (Abcam), Best1 IgG at 1:100] and incubated overnight at $4^{\circ} \mathrm{C}$. After this, the sections were washed three times in $0.1 \mathrm{M} \mathrm{PBS}$ and incubated in secondary antibody [DyLight 488 donkey anti-chicken IgG at 1:200 (The Jackson Laboratory), Alexa 555 donkey anti-goat IgG at 1:200 (Invitrogen)] for $2 \mathrm{~h}$. After three rinses in 0.1 M PBS and DAPI staining at 1:1000 (PIERCE), the sections were mounted on polysine microscopic slide glass (Thermo Scientific). Images were acquired using a Nikon A1R confocal microscope.

\section{Statistical analysis}

Statistical analysis was performed using Prism software (GraphPad software). Statistical significance between two groups was analyzed by using student t-test and among multiple groups at least three groups by using One-way ANOVA with Dunnett's multiple comparison. All values are shown as mean \pm s.e.m. 


\section{Competing interests}

The authors declare that they have no competing interests.

\section{Authors' contributions}

HP performed and designed the experiments, performed a whole-cell voltage clamp of hippocampal CA1 astrocytes, and wrote the manuscript. KSH performed a dual patch clamp onto hippocampal astrocytes, and wrote the manuscript. SJO performed a whole cell patch clamp recording for SK1 channel. JS performed a whole-cell voltage clamp of hippocampal CA1 astrocytes. BEY and SJ performed immunohistochemistry. CJL designed the experiments and wrote the manuscript, and supervised the entire project. All authors read and approved the final manuscript.

\section{Acknowledgements}

This work was supported by the World Class Institute (WCI 2009-003) of the National Research Foundation (NRF) funded by the Korean Ministry of Science and Technology (MEST), and National Agenda Project (NAP) of the Korea Research Council of Fundamental Science \& Technology.

\section{Author details}

${ }^{1}$ Center for Neural Science and WCI Center for Functional Connectomics, Korea Institute of Science and Technology (KIST), Seoul, Korea. ${ }^{2}$ Neuroscience Program, University of Science and Technology (UST), Daejeon, Korea. ${ }^{3}$ KU-KIST Graduate School of Converging Science \& Technology, Seoul, Korea. ${ }^{4}$ Department of Biological Science, Korea Advanced Institute of Science and Technology (KAIST), Daejeon, Korea. ${ }^{5}$ Department of Nanobiomedical Science, Dankook University, Cheonan, Korea.

Received: 17 August 2013 Accepted: 4 December 2013 Published: 9 December 2013

\section{References}

1. Haydon PG, Carmignoto G: Astrocyte control of synaptic transmission and neurovascular coupling. Physiol Rev 2006, 86(3):1009-1031.

2. Halassa MM, Fellin T, Haydon PG: The tripartite synapse: roles for gliotransmission in health and disease. Trends Mol Med 2007, 13(2):54-63.

3. Lee CJ, Mannaioni G, Yuan H, Woo DH, Gingrich MB, Traynelis SF: Astrocytic control of synaptic NMDA receptors. J Physiol 2007, 581(Pt 3):1057-1081.

4. Woo DH, Han KS, Shim JW, Yoon BE, Kim E, Bae JY, Oh SJ, Hwang EM, Marmorstein AD, Bae YC, et al: TREK-1 and Best1 channels mediate fast and slow glutamate release in astrocytes upon GPCR activation. Cell 2012, 151(1):25-40.

5. Han KS, Woo J, Park H, Yoon BJ, Choi S, Lee CJ: Channel-mediated astrocytic glutamate release via Bestrophin-1 targets synaptic NMDARs. Mol Brain 2013, 6(1):4.

6. Lee S, Yoon BE, Berglund K, Oh SJ, Park H, Shin HS, Augustine GJ, Lee CJ: Channel-mediated tonic GABA release from glia. Science 2010, 330(6005):790-796.

7. Park H, Oh SJ, Han KS, Woo DH, Park H, Mannaioni G, Traynelis SF, Lee CJ: Bestrophin-1 encodes for the Ca2 + -activated anion channel in hippocampal astrocytes. J Neurosci 2009, 29(41):13063-13073.

8. Qu Z, Hartzell HC: Bestrophin $\mathrm{Cl}$ - channels are highly permeable to $\mathrm{HCO}$. Am J Physiol Cell Physiol 2008, 294(6):C1371-C1377.

9. O'Driscoll KE, Leblanc N, Hatton WJ, Britton FC: Functional properties of murine bestrophin 1 channel. Biochem Biophys Res Commun 2009, 384(4):476-481.

10. Zhou M, Xu G, Xie M, Zhang X, Schools GP, Ma L, Kimelberg HK, Chen H: TWIK-1 and TREK-1 are potassium channels contributing significantly to astrocyte passive conductance in rat hippocampal slices. J Neurosci 2009, 29(26):8551-8564.

11. Takano T, Kang J, Jaiswal JK, Simon SM, Lin JH, Yu Y, Li Y, Yang J, Dienel G, Zielke HR, et al: Receptor-mediated glutamate release from volume sensitive channels in astrocytes. Proc Natl Acad Sci U S A 2005, 102(45):16466-16471.

12. Qu Z, Fischmeister R, Hartzell C: Mouse bestrophin-2 is a bona fide Cl(-) channel: identification of a residue important in anion binding and conduction. J Gen Physiol 2004, 123(4):327-340.

13. Davidson JS, Baumgarten IM, Harley EH: Reversible inhibition of intercellular junctional communication by glycyrrhetinic acid. Biochem Biophys Res Commun 1986, 134(1):29-36.
14. Spray DC, Rozental R, Srinivas M: Prospects for rational development of pharmacological gap junction channel blockers. Curr Drug Targets 2002, 3(6):455-464.

15. Saez JC, Contreras JE, Bukauskas FF, Retamal MA, Bennett MV: Gap junction hemichannels in astrocytes of the CNS. Acta Physiol Scand 2003, 179(1):9-22

16. Suadicani SO, Brosnan CF, Scemes E: P2X7 receptors mediate ATP release and amplification of astrocytic intercellular Ca2+ signaling. J Neurosci 2006, 26(5):1378-1385.

17. Hille B: Ion Channels of Excitable Membranes. 3rd edition. Sunderland, Mass: Sinauer; 2001.

18. Bar-Yehuda D, Korngreen A: Space-clamp problems when voltage clamping neurons expressing voltage-gated conductances. J Neurophysiol 2008, 99(3):1127-1136.

19. Armstrong CM, Gilly WF: Access resistance and space clamp problems associated with whole-cell patch clamping. Methods Enzymol 1992, 207:100-122.

20. Augustine GJ, Charlton MP, Smith SJ: Calcium entry and transmitter release at voltage-clamped nerve terminals of squid. J Physiol 1985, 367:163-181.

21. Johnston D, Magee JC, Colbert CM, Cristie BR: Active properties of neuronal dendrites. Annu Rev Neurosci 1996, 19:165-186.

22. Larsson HP, Kleene SJ, Lecar H: Noise analysis of ion channels in nonspace-clamped cables: estimates of channel parameters in olfactory cilia. Biophysi J 1997, 72(3):1193-1203.

23. Muller W, Lux HD: Analysis of voltage-dependent membrane currents in spatially extended neurons from point-clamp data. J Neurophysio/ 1993, 69(1):241-247

24. Spruston N, Jaffe DB, Williams SH, Johnston D: Voltage- and space-clamp errors associated with the measurement of electrotonically remote synaptic events. J Neurophysiol 1993, 70(2):781-802.

25. White JA, Sekar NS, Kay AR: Errors in persistent inward currents generated by space-clamp errors: a modeling study. J Neurophysiol 1995, 73(6):2369-2377.

26. Ormel L, Stensrud MJ, Bergersen LH, Gundersen V: VGLUT1 is localized in astrocytic processes in several brain regions. Glia 2012, 60(2):229-238.

27. Rall W: Time constants and electrotonic length of membrane cylinders and neurons. Biophysic J 1969, 9(12):1483-1508.

28. Ventura A, Meissner A, Dillon CP, McManus M, Sharp PA, Van Parijs L, Jaenisch R, Jacks T: Cre-lox-regulated conditional RNA interference from transgenes. Proc Natl Acad Sci U S A 2004, 101(28):10380-10385.

29. Cetin A, Komai S, Eliava M, Seeburg PH, Osten P: Stereotaxic gene delivery in the rodent brain. Nat Protoc 2006, 1(6):3166-3173.

30. Kafitz KW, Meier SD, Stephan J, Rose CR: Developmental profile and properties of sulforhodamine 101-Labeled glial cells in acute brain slices of rat hippocampus. J Neurosci Methods 2008, 169(1):84-92.

doi:10.1186/1756-6606-6-54

Cite this article as: Park et al:: High glutamate permeability and distal localization of Best1 channel in CA1 hippocampal astrocyte. Molecular Brain $20136: 54$.

\section{Submit your next manuscript to BioMed Central and take full advantage of:}

- Convenient online submission

- Thorough peer review

- No space constraints or color figure charges

- Immediate publication on acceptance

- Inclusion in PubMed, CAS, Scopus and Google Scholar

- Research which is freely available for redistribution 